(C) В.И.Помазкин, 2017

УДК 616.345-006-06:616.345-007.272-089

\title{
В.И. Помазкин
}

\section{СРАВНИТЕЛЬНЫЕ РЕЗУЛЬТАТЫ ВОССТАНОВИТЕЛЬНОГО ЭТАПА ПРИ ДВУХЭТАПНОЙ ТАКТИКЕ ЛЕЧЕНИЯ ОПУХОЛЕВОЙ ТОЛСТОКИШЕЧНОЙ НЕПРОХОДИМОСТИ}

\begin{abstract}
Целью исследования являлся сравнительный анализ результатов при разных подходах к двухэтапному лечению опухолевой толстокишечной непроходимости на этапе восстановления целостности кишечного тракта. В основную группу включено 260 больных с фрормированием на первом этапе двуствольной колостомы и последующей резекцией толстой кишки с удалением опухоли и ликвидацией стомы. В контрольную группу включено 192 больных с выполнением на первом этапе обструктивной резекции толстой кишки и последующей восстановительной операции. Интраоперационные повреждения тонкой кишки отмечены у 6,9\% больных основной группы и у 18,2\% пациентов контрольной группы. Послеоперационная летальность в основной группе составила 1,2\%, в контрольной группе - 1,5\%. Ранние послеоперационные осложнения наблюдались у 9,2\% и 17,7\% пациентов соответственно. Основным фрактором риска возникновения осложнений в контрольной группе был выраженный спаечный процесс брюшной полости. Сделан вывод о преимуществах для ликвидации опухолевой толстокишечной непроходимости формирования колостомы на первом этапе и выполнения радикальной операции на втором этапе лечения.
\end{abstract}

Ключевые слова: рак толстой кишки, кишечная непроходимость, колостома, операция Гартмана

\section{V.I.Pomazkin}

\section{COMPARATIVE RESULTS OF RECOVERY STAGE IN TWO-STEP TREATMENT OF MALIGNANT COLONIC OBSTRUCTION}

Sverdlovsk regional clinical hospital for war veterans, Yekaterinburg

The aim of the study was a comparative analysis of results of different approaches to two-stage treatment of malignant colonic obstruction on the stage of recovery of the integrity of intestinal tract. The main group included 260 patients. A double-barreled colostomy was formed at the first stage, than resection of the colon with tumor removing and stoma excision were performed. The control group consisted of 192 patients. An obstructive resection of the colon was made at the first stage with following reconstructive operation. Intraoperative damage of the small intestine was observed in $6,9 \%$ patients of the main group and $18,2 \%$ patients of the control group. Postoperative mortality consisted of $1,2 \%$ in the main group and it was $1,5 \%$ in the control group. The early postoperative complications numbered $9,2 \%$ and $17,7 \%$, respectively. The main risk factor of complication development was an expressed adhesion process of the abdominal cavity in the control group of patients. CONCLUSIONS. The method, which included the colostomy formation at the first stage with following radical surgery at the second stage, had advantages in case of elimination of malignant colonic obstruction.

Key words: colon cancer, intestinal obstruction, colostomy, Hartmann's surgery

В в еде ни е . Актуальность лечения опухолевой обтурационной толстокишечной непроходимости обусловлена неуклонным ростом частоты этой патологии, а также сохраняющейся нерешенностью и спорностью выбора оптимальной тактики оперативного лечения. Особенно это касается кишечной непроходимости при стенозирующем раке левой половины толстой кишки. Варианты лечения включают как одномоментные операции с удалением опухоли и первичным восстановлением целостности кишечного тракта, так и многоэтапное лечение, в том числе с использованием эндоскопических внутрикишечных процедур и саморасширяющихся внутрипросветных стентов.

Несмотря на многообразие подходов, наиболее распространенной при опухолевой толстокишечной непроходимости остается двухэтапная тактика, включающая выполнение обструктивной резекции толстой кишки на первом этапе, а затем восстановительную операцию с ликвидацией одноствольной колостомы. Однако 
число осложнений при выполнении радикальной операции у больных, находящихся в тяжелом состоянии вследствие кишечной непроходимости, остается достаточно большим, достигая $55 \%$ $[8,14]$. Летальность при выполнении неотложной операции Гартмана составляет от 5 до $35 \%$ [2, 3, 8]. Для уменьшения риска возникновения послеоперационных осложнений предложено использовать тактику, включающую формирование двуствольной колостомы проксимальнее уровня обтурации толстой кишки, а затем после ликвидации явлений непроходимости выполнение радикальной операции в плановом порядке с восстановлением пассажа по толстой кишке. Уменьшение объема первичного вмешательства снижает частоту ранних осложнений и послеоперационную смертность при ликвидации кишечной непроходимости даже у пациентов с высоким операционным риском $[1,2,5]$. Остается открытым вопрос о преимуществах того или иного варианта хирургической тактики при восстановительном оперативном лечении для улучшения радикальности лечения и получения оптимальных послеоперационных результатов.
Материал и методы . В ретророспективное исследование включены непосредственные результаты оперативного лечения 452 больных с декомпенсированной непроходимостью при стенозирующем раке левой половины толстой кишки, оперированных в период с 2007 по 2015 г. Критериями исключения из исследования являлись пациенты с генерализацией опухолевого процесса перед восстановительной операцией.

Основную группу составили 260 больных с формированием на первом этапе с целью ликвидации кишечной непроходимости двуствольной колостомы. После купирования явлений непроходимости, нормализации состояния этим пациентам в плановом порядке выполняли радикальную резекцию толстой кишки с удалением опухоли и одномоментным восстановлением пассажа по толстой кишке. В контрольную группу включены 192 больных с выполнением на первом этапе обструктивной резекции толстой кишки и формированием одноствольной колостомы. На втором этапе лечения в этой группе производили восстановительную операцию с ликвидацией стомы и восстановлением целостности толстой кишки. Для сопоставимости сравнительных результатов в основную группу не включали пациентов с местно-распространенным опухолевым процессом, требующим комбинированных вмешательств на нескольких органах, а в контрольную группу - больных с местным рецидивом опухоли после операции Гартмана, выявленным к моменту восстановительной операции, также требующих расширения объема оперативного вмешательства.

Характеристика пациентов, включенных в исследование

\begin{tabular}{|c|c|c|}
\hline Параметры & Основная группа, $n=260$ & Контрольная группа, n=192 \\
\hline Средний возраст, лет & $67,3 \pm 11,2$ & $65,2 \pm 10,2$ \\
\hline Соотношение мужчины/женщины & $110 / 150$ & $85 / 107$ \\
\hline \multicolumn{3}{|l|}{ Стадия опухолевого процесса: } \\
\hline II & $173(66,5 \%)$ & $139(72,4 \%)$ \\
\hline IIIB & $65(25,0 \%)$ & $46(24,0 \%)$ \\
\hline IIIC & $22(8,5 \%)^{*}$ & $7(3,6 \%)$ \\
\hline Интервал между операциями, дни & $41,2 \pm 9,1^{*}$ & $196,3 \pm 39,2$ \\
\hline \multicolumn{3}{|l|}{ Вид колостомы: } \\
\hline трансверзостома & $98(37,7 \%)$ & $65(33,9 \%)$ \\
\hline сигмостома & $162(62,4 \%)$ & $127(66,1 \%)$ \\
\hline \multicolumn{3}{|l|}{ Вид радикальной операции: } \\
\hline левосторонняя гемиколэктомия & $113(43,5 \%)^{\star}$ & $65(33,9 \%)$ \\
\hline резекция сигмовидной кишки & $95(36,5 \%)^{*}$ & $91(47,4 \%)$ \\
\hline резекция прямой кишки & $52(20 \%)$ & $36(18,7 \%)$ \\
\hline «Короткая» культя прямой кишки & $35(13,5 \%)$ & $29(15,1 \%)$ \\
\hline \multicolumn{3}{|c|}{ Выраженность спаечного процесса брюшной полости } \\
\hline Отсутствие спаек, степень: & $118(45,4 \%)^{*}$ & $10(5,2 \%)$ \\
\hline I & $94(36,2 \%)^{*}$ & $88(45,8 \%)$ \\
\hline II & $36(16,5 \%)^{*}$ & $51(26,6 \%)$ \\
\hline III & $12(4,6 \%)^{*}$ & $43(22,4 \%)$ \\
\hline
\end{tabular}

Здесь и в табл. 2-3: p<0,05. 
Bce операции на первом этапе выполняли в различных общехирургических городских и районных стационарах Свердловской области, оказывающих неотложную помощь. В основной группе у $176(67,7 \%)$ пациентов разгрузочную колостому формировали из мини-доступа, у 84 (32,4\%) больных использовали срединную лапаротомию. В контрольной группе подобный доступ был выполнен у всех пациентов. Все восстановительные операции выполняли в специализированном отделении «Свердловского областного госпиталя для ветеранов войн» врачи-колопроктологи.

Группы пациентов были сопоставимы по возрасту, половому составу, сопутствующей патологии, стадии опухолевого процесса (табл. 1). Все восстановительные операции были выполнены из срединного лапаротомного доступа. Дистальная культя прямой кишки расценивалась как «короткая» при длине менее 10 см и расположении ее ниже тазовой брюшины либо при необходимости ее резекции ниже этого уровня в основной группе.

Интервал между операциями в основной группе составил $(41,2 \pm 9,1)$ дня, в контрольной - $(196,3 \pm 39,2)$ дня. Отличием у больных основной группы являлось более частое выполнение левосторонней гемиколэктомии. Самым существенным различием между группами была выраженность спаечного процесса в брюшной полости в областях, удаленных от сформированной колостомы. Если в основной группе у 118 $(45,4 \%)$ больных спаек не было, то в контрольной группе спаечные сращения отсутствовали лишь у 10 (5,2\%) пациентов.

Учитывая, что спаечный процесс может существенно влиять на технические особенности операции, мы производили его оценку с градацией по 3 степеням с учетом макроморфологических характеристик: I степень - мягкие пленчатые эластичные спайки, легко поддающиеся растяжению с возможностью их разделения тупым способом; II степень - более плотные, ограниченно растяжимые плоскостные спайки, при попытке тупого их разделения возможно травмирование серозной оболочки, что требовало висцеролиза острым путем; III степень - плотные, нерастяжимые плоскостные васкуляризованные сращения, деформирующие кишку, требующие только острого разделения. Если в контрольной группе спаечный процесс II и III степени присутствовал у $49 \%$ больных, то в основной группе - лишь у 21,1\% пациентов.

Статистическую обработку полученных данных осуществляли с помощью программы Statistica 6.0 (StatSoft, Inc., США). Для сравнительной оценки категориальных переменных использовали критерий $\chi^{2}$ Пирсона и точный метод Фишера. Тест t Стьюдента использовали для сравнения количественных непрерывных переменных. Для оценки вероятности возникновения осложнений использовали измерение отношения рисков с 95\% доверительным интервалом (ДИ) Различие между исследуемыми группами считалось статистически достоверным при $\mathrm{p}<0,05$.

Результаты. В качестве косвенного критерия оценки технической сложности выполнения операции использовали среднее время от начала разреза кожи до начала основного этапа. В основной группе это было время до начала мобилизации толстой кишки с опухолью и стомированным участком, в контрольной группе - время до выделения либо дистальной культи толстой кишки, либо время до выделения стомированного участка из брюшной стенки. В основной группе среднее время составило $(9,5 \pm 4,1)$ мин (диапазон от 5 до 30 мин), в контрольной - $(31,3 \pm 17,2)$ мин (от 8 до 85 мин) $(\mathrm{p}<0,01)$. Основной причиной значительного увеличения времени были затруднения при входе в брюшную полость и адгезиолизисе с расправлением петель тонкой и толстой кишки вследствие спаечного процесса. При этом время операции значительно возрастало при плотных распространённых внутрибрюшных сращениях.

Основным интраоперационным осложнением было повреждение тонкой кишки при адгезиолизисе. Множественные участки десерозирования кишки отмечены у 18 (6,9\%) больных основной группы и у $35(18,2 \%)$ пациентов контрольной группы (р<0,01). При этом сквозные повреждения тонкой кишки возникли у $7(2,7 \%)$ и $16(8,3 \%)$ пациентов $(\mathrm{p}<0,01)$ соответственно. Все замеченные участки повреждений кишки были ушиты. У $6(3,1 \%)$ больных контрольной группы в связи с множественным характером повреждений, расположенных на ограниченном участке с грубыми рубцовыми изменениями тонкой кишки, выполнена ее резекция. Размер удаляемой ее части составил от 12 до 50 см. Подобное расширение операции в основной группе потребовалось только у $1(0,4 \%)$ пациентки.

Несмотря на примерно равную частоту «короткой культи» прямой кишки, технические трудности при ее выделении при подготовке к анастомозированию наблюдались у 15 (7,5\%) больных после ранее выполненной операции Гартмана. Это было связано с развитием плотных рубцовых изменений тазовой брюшины, плотными сращениями между культей прямой кишки и тазовыми органами. Интраоперационное повреждение мочевого пузыря при этом наблюдалось в $2(1 \%)$ случаях, сквозное повреждение стенки влагалища - у $1(0,5 \%)$ больной, повреждение пресакрального венозного сплетения - у $1(0,5 \%)$ пациента. В основной группе резекция прямой кишки не отличалась технической сложностью и проходила без повреждения прилежащих тазовых органов и структур.

Непосредственные результаты оценивали в срок 30 дней после операции. Послеоперационная летальность в основной группе составила $1,2 \%$, в контрольной группе $-1,5 \%$. Ранние послеоперационные осложнения отражены в табл. 2 . В основной группе осложнения наблюдались у 24 $(9,2 \%)$ больных, в контрольной - у $34(17,7 \%)$ пациентов $(\mathrm{p}<0,01)$. Повторные оперативные вмешательства потребовались у $5(1,9 \%)$ и $9(4,6 \%)$ больных $(\mathrm{p}<0,01)$ соответственно. В основной группе достоверно реже наблюдались отсрочен- 
ные перфорации тонкой кишки и менышая частота возникновения длительного пареза желудочнокишечного тракта, критериями которого являлись задержка отхождения стула и газов, необходимость назогастрального дренирования более 4 сут.

Унивариантный анализ отношения рисков возникновения послеоперационных осложнений показал, что единственным достоверным фактором их увеличения в основной группе являлось выполнение левосторонней гемиколэктомии (maбл. 3). При ней в сравнении с резекцией сигмовидной кишки риск возможных осложнений возрастал в 2,5 раза $(\mathrm{p}<0,05)$.

В контрольной группе существенными факторами риска являлись наличие гнойных осложнений при проведении операции Гартмана на первом этапе лечения непроходимости и особенно степень выраженности спаечного процесса в брюшной полости. Гнойно-воспалительные осложнения на предыдущем этапе лечения увеличивали риск возникновения осложнений при восстановительной операции в 4,3 раза ( $\mathrm{p}<0,01)$, III степень выраженности спаечного процесса в сравнении со II степенью приводила к увеличению в 4,5 раза $(\mathrm{p}<0,01)$, а в сравнении с I степенью - в 9,7 раза $(\mathrm{p}<0,001)$.

О б с у ж д е н и е . Очевидными преимуществами тактики лечения опухолевой толстокишечной непроходимости с формированием колостомы на первом этапе в сравнении с операцией Гартмана является быстрое обеспечение декомпрессии кишки с минимальной хирургической травмой, что позволяет уменьшить риск неблагоприятного исхода у больных, находящихся в тяжелом состоянии. После ликвидации кишечной непроходимости существует возможность полноценного
Таблица 2

Ранние послеоперационные осложнения при восстановительных операциях

\begin{tabular}{|c|c|c|}
\hline Осложнение & Основная группа & Контрольная группа \\
\hline $\begin{array}{l}\text { Несостоятельность } \\
\text { колоректального } \\
\text { анастомоза }\end{array}$ & $3(1,2 \%)$ & $3(1,6 \%)$ \\
\hline $\begin{array}{l}\text { Отсроченная } \\
\text { перфоорация } \\
\text { тонкой кишки }\end{array}$ & $1(0,4 \%)^{*}$ & $3(1,6 \%)$ \\
\hline $\begin{array}{l}\text { Внутрибрюшное } \\
\text { кровотечение }\end{array}$ & - & $2(1,0 \%)$ \\
\hline $\begin{array}{l}\text { Инсрильтрат брюшной } \\
\text { полости }\end{array}$ & $2(0,8 \%)$ & $3(1,6 \%)$ \\
\hline $\begin{array}{l}\text { Длительный парез } \\
\text { кишечного тракта }\end{array}$ & $7(2,7 \%)^{*}$ & $15(7,8 \%)$ \\
\hline $\begin{array}{l}\text { Формирование } \\
\text { тонкокишечного } \\
\text { свища }\end{array}$ & $1(0,4 \%)$ & $1(0,5 \%)$ \\
\hline $\begin{array}{l}\text { Нагноение } \\
\text { лапаротомной раны }\end{array}$ & $4(1,5 \%)$ & $4(2,1 \%)$ \\
\hline $\begin{array}{l}\text { Нагноение } \\
\text { раны в месте } \\
\text { существования } \\
\text { колостомы }\end{array}$ & $6(2,3 \%)$ & $3(1,5 \%)$ \\
\hline Всего & $24(9,2 \%)^{*}$ & $34(17,7 \%)$ \\
\hline
\end{tabular}

обследования толстой кишки и подготовки пациента к радикальной операции $[1,2,5]$. Важным преимуществом такой тактики является сокращение времени существования колостомы, что улучшает качество жизни пациентов.

Восстановление целостности кишечного тракта после выполнения обструктивной резекции толстой кишки с удалением опухоли, несмотря на кажущуюся простоту по замыслу, сталкивается с серьезными техническими трудностями. О слож-

Унивариантный анализ факторов риска, ассоциированных с послеоперационными осложнениями

\begin{tabular}{l|c|c}
\hline \multicolumn{1}{c|}{ Параметры } & $\begin{array}{c}\text { Отношение рисков } \\
\text { в основной группе (ДИ 95\%) }\end{array}$ & $\begin{array}{c}\text { Отношение рисков } \\
\text { в контрольной группе (Ди 95\%) }\end{array}$ \\
\hline Возраст (менее 60 лет или более 60 лет) & $2,3(0,14-3,3)$ & $1,2(0,13-6,52)$ \\
Пол (мужчины или женщины) & $4,1(0,1-6,5)$ & $2,3(0,3-4,5)$ \\
Тип колостомы (трансверзостома или сигмостома) & $2,2(0,05-2,8)$ & $4,5(0,05-3,4)$ \\
Стадия опухолевого процесса, II или III & $2,1(0,4-4,5)$ & $3,3(0,2-5,6)$ \\
Вид радикальной операции (левосторонняя гемиколэктомия & $2,5(1,5-4,4)^{*}$ & $2,1(0,3-4,7)$ \\
или резекция сигмовидной кишки) & \\
Гнойные осложнения при первичном вмешательстве & $1,1(0,2-1,5)$ & $4,3(1,7-11,3)^{*}$ \\
(наличие или отсутствие) & & \\
Короткая культя прямой кишки (наличие или отсутствие) & $1,4(0,08-2,3)$ & $0,28(0,08-1,00)$ \\
Выраженность спаечного процесса (III или I степень) & $2,2(0,54-4,2)$ & $9,7(2,54-24,1)^{*}$ \\
Выраженность спаечного процесса (III или II степень) & $1,5(0,3-2,6)$ & $4,5(1,3-8,6)^{*}$
\end{tabular}


ности восстановительных операций на толстой кишке свидетельствует высокий уровень интраоперационных и послеоперационных осложнений, по данным различных авторов составляющий от 5,6 до $60 \%$, с летальностью до $3 \%$ [3, 9, 12]. Во многом с этим связана высокая частота отказов пациентам в восстановительном вмешательстве после операции Гартмана, достигающая 50\% случаев, при которых колостома, планируемая на этапе неотложной операции как временная, превращается в постоянную $[3,8,13]$.

В нашем исследовании при оценке восстановительного этапа лечения при опухолевой непроходимости операция с радикальным удалением опухоли и ликвидацией колостомы, несмотря на больший объем вмешательства, часто технически оказывалась проще для выполнения по сравнению с восстановлением целостности кишечного тракта после операции Гартмана. При этом число послеоперационных осложнений оказалось большим в группе пациентов с ранее выполненной резекцией толстой кишки.

Основной причиной этого являлся спаечный процесс брюшной полости, выраженное развитие которого приводило к трудностям при осуществлении висцеролиза с идентификацией и выделением тонкой и толстой кишки. В ряде работ спаечный процесс брюшной полости оценивается как существенный предиктор возникновения послеоперационных осложнений в колоректальной хирургии [6, 11]. Развитие множественных спаечных сращений приводит к удлинению операции, увеличению частоты конверсий при попытке выполнения лапароскопических вмешательств, существенному увеличению частоты интраоперационных повреждений тонкой и толстой кишки и мочевыводящих путей при адгезиолизисе, возрастанию числа последующих осложнений $[11,13]$.

В нашем исследовании степень выраженности спаечного процесса коррелировала с частотой послеоперационных осложнений. Учитывая техническую сложность восстановительной операции при одноствольной колостоме, высокую частоту послеоперационных осложнений и частые отказы в ее проведении с превращением временной стомы в постоянную, вероятно актуальными являются предложения ряда авторов при отказе от выполнения операции Гартмана в качестве первого этапа лечения опухолевой кишечной непроходимости.

Одним из вариантов является использование интракишечных вмешательств, в частности установка в зону опухолевого сужения саморасширяющихся стентов, направленных на устранение явлений острой кишечной непроходимости, с выполнением последующего радикального вмешательства [4]. Самым существенным недостатком, ограничивающим широкое внедрение такого перспективного метода лечения, является высокая стоимость стентов, а также возможные осложнения.

Другим вариантом является выполнение неотложной радикальной операции с наложением первичного толстокишечного анастомоза $[5,7,9]$. Однако такие операции, как правило, выполнимы в крупных клиниках с соответствующим техническим и кадровым обеспечением и определенным отбором пациентов. Учитывая то, что неотложная помощь при обтурацинной опухолевой толстокишечной непроходимости оказывается в общехирургических стационарах, актуальными, по нашему мнению, становятся формирование разгрузочной колостомы из мини-доступа и затем выполнение радикального оперативного вмешательства, наряду с ликвидацией колостомы, в специализированном стационаре.

В ы в оды . 1. При двухэтапной тактике лечения опухолевой обтурационной толстокишечной непроходимости на этапе восстановительного вмешательства выполнение радикальной операции с ликвидацией колостомы приводит к менышему числу осложнений, чем восстановление кишечного тракта после ранее выполненной операции Гартмана.

2. Восстановительные операции после обструктивной резекции толстой кишки технически являются более сложным вмешательством, что связано с выраженностью спаечного процесса в брюшной полости, приводящего к удлинению времени проведения операции и увеличению числа интраоперационных осложнений.

\section{ЛИТЕРАTУPA [REFERENCE]}

1. Помазкин В.И., Мансуров Ю.В. Тактика оперативного лечения при опухолевой обтурационной толстокишечной непроходимости // Хирургия. 2008. № 9. С. 15-18 [Pomazkin V.I., Mansurov Ju.V. Taktika operativnogo lechenija pri opuholevoj obturacionnoj tolstokishechnoj neprohodimosti // Hirurgija. 2008. № 9. P. 15-18].

2. Пугаев А.В., Ачкасов Е.Е. Обтурационная опухолевая толстокишечная непроходимость. М.: ПРОФИЛЬ, 2005. 224 с. [Pugaev A.V., Achkasov E.E. Obturacionnaja opuholevaja tolstokishechnaja neprohodimost'. M.: PROFIL", 2005. 224 p.].

3. Banerjee S., Leather A., Rennie J. et al. Feasibility and morbidity of reversal of Hartmann's // Colorectal Dis. 2005. Vol. 7. P. 454-459.

4. Berg M., Sloothaak D., Dijkgraaf M. et al. Bridge-to-surgery stent placement versus emergency surgery for acute malignant colonic obstruction // Br. J. Surg. 2014. Vol. 101. P. 807-873.

5. Chereau N., Lefevre J., Lefrancois M. et al. Management of malignant left colonic obstruction: is an initial temporary colostomy 
followed by surgical resection a better option? // Colorectal Dis. 2013. Vol. 15. P. e646-e653.

6. Coleman M., McLain A., Moran B. Impact of previous surgery on time taken for incision and division of adhesions during laparotomy // Dis. Colon Rectum. 2000. Vol. 43. P. 1297-1299.

7. Cross K.L., Rees J., Soulsby R., Dixon A. Primary anastomosis without colonic lavage for the obstructed left colon // Ann. R. Coll. Surg. Engl. 2008. Vol. 90. P. 302-304.

8. Deans G., Krukowski Z., Irwin S. Malignant obstruction of the left colon // Br. J. Surg. 2005. Vol. 81. P. 1270-1276.

9. Garber A., Hyman N., Osler T. Complications of Hartmann takedown in a decade of preferred primary anastomosis // Am. J. Surg. 2014. Vol. 207. P. 60-64.
10. Frago R., Ramirez E., Milan M. et al. Current management of acute malignant large bowel obstruction: a systematic review // Am. J. Surg. 2014. Vol. 207. P. 127-138.

11. Parker M., Wilson M., Menzies D. et al. Colorectal surgery: the risk and burden of adhesion-related complications // Colorectal Dis. 2004. Vol. 6. P. 506-511.

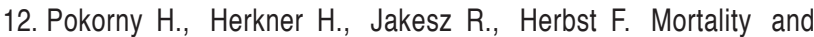
complications after stoma closure //Arch. Surg. 2005. Vol. 140. P. 956-960.

13. Roig J., Cantos M,, Balciscueta Z. et al. Hartmann's operation: how often is it reversed and at what cost? A multicentre study // Colorectal Dis. 2011. Vol. 13. P. e396-e402.

14. Smothers L., Hynan L., Fleming J. et al. Emergency surgery for colon carcinoma // Dis. Colon Rectum. 2003. Vol. 46. P. 24-30.

Поступила в редакцию 21.06.2016 г.

\section{Сведения об авторе:}

Помазкин Вадим Игоревич (e-mail: yunker@yandex.ru), канд. мед. наук, Свердловский областной клинический госпиталь для ветеранов войн 620036, г. Екатеринбург, ул. Соболева, 25 